\title{
Anti-Prostate Cancer Activity of Plant-Derived Bioactive Compounds: a Review
}

\author{
Cindy Thomas-Charles ${ }^{1} \cdot$ Herman Fennell $^{1}$
}

Published online: 17 July 2019

(C) The Author(s) 2019

\begin{abstract}
Purpose of Review Prostate cancer is one of the most common cancers in men and accounts for about $10 \%$ of all new cancer cases in the USA. Despite significant improvements in survival, it is estimated that deaths from prostate cancer in 2019 will exceed 30,000 individuals. Here, we review plant-derived bioactive compounds with the ability to modulate the growth of prostate cancer cells. These compounds represent potential therapeutic alternatives for the prevention and treatment of prostate cancer. Recent Findings Numerous plants produce phytochemicals that are important for their development and protection. Many of these compounds have inhibitory effects on the growth of cancer cells.

Summary Cancers are a leading cause of death worldwide and treatments tend to be costly with many negative side effects. Identification of new potential chemo-therapeutic and chemo-protective compounds that have little or no negative effects on normal cells is therefore of great importance.
\end{abstract}

Keywords Prostate cancer $\cdot$ Cytotoxicity $\cdot$ Phytochemicals $\cdot$ Anticancer $\cdot$ Bioactive compounds

\section{Introduction}

According to the Centers for Disease Control and Prevention, prostate cancer remains one of the most common cancers among men of all races second only to non-melanoma skin cancer (https://www.cdc.gov/cancer/prostate/statistics/index. htm). The Surveillance, Epidemiology, and End Results Program at the National Cancer Institute indicates that the estimated new cases of prostate cancer in the USA in 2019 will be nearly 200,000 individuals, and account for almost $10 \%$ of all new cancer cases. It is further estimated that about 30,000 men will die from prostate cancer in 2019. The observed rate of prostate cancer has declined in the USA by more than half between 1992 and 2016. Additionally, the 5year relative survival rate has increased from $66.3 \%$ in 1975 to

This article is part of the Topical Collection on Molecular Biology of Prostate Cancer

Herman Fennell

herman.fennell@hamptonu.edu

Cindy Thomas-Charles

cindy.thomascharles@gmail.com

1 Hampton University, 200 William R. Harvey Way, Hampton, VA 23668, USA
99.2\% in 2010 (https://seer.cancer.gov/statfacts/html/prost. $\underline{\mathrm{html}}$ ). Perhaps, most alarming is the observation that prostate cancers have the ability to metastasize to the bone. When this occurs, the 5-year survival rate of patients drops to less than $1 \%$ [1]. Despite the overall decreased rate of prostate cancer in the USA, there exists a cancer health disparity where African-American males are twice as likely to be diagnosed with and die from prostate cancer compared to Caucasian men. The precise cause of this disparity is not well understood, but it is generally accepted that socioeconomic behavioral factors, such as access to affordable healthcare and diet, and molecular factors may all play a significant role $[2,3]$. Interestingly, it has been suggested that as much as a third of the annual deaths in the USA due to chronic illnesses, including cancer, could potentially be avoided by dietary modifications [4].

The specific combination of therapies currently available for the treatment of prostate cancer tends to be based on the advancement of cancer. For example, radical prostatectomy, which is the complete removal of the prostate and associated tissue, is typically combined with radiation therapy for the treatment of low-grade prostate cancers. While androgen deprivation therapy is used to treat cancers that have spread beyond the boundaries of the gland, have reoccurred, or in cases where shrinking the tumor prior to surgery is believed to be more beneficial to the patient. Hormone therapy alone does 
not cure prostate cancer. Instead, the goal of hormonal therapy is usually to reduce or stop the growth of the prostate cells by depriving them of the two main androgens produced by the testicles - testosterone and dihydrotestosterone. Combinations of hormone and chemotherapy are often used for cancers that are more advanced or have metastasized. According to the American Cancer Society, some of the commonly used chemotherapeutic drugs include docetaxel that inhibits microtubule assembly and subsequently cell division; cabazitaxel, a derivative of docetaxel [5]; mitoxantrone, a topoisomerase inhibitor [6]; and estramustine, which is also an antimicrotubule agent [7]. Not surprisingly, there are a number of generalized side effects associated with the use of these drugs. These include reduced red and white blood cell count, which causes the patient to develop fatigue, and increased chance of infection respectively. More severe side effects of treatment with these drugs are peripheral neuropathy, increased risk of developing blood clots, and in rare cases, development of leukemia [7] (https://www.cancer.org/cancer/prostate-cancer/treating/ chemotherapy.html).

\section{Common Models of Prostate Cancer}

Recent studies that assessed the effects of phytochemicals on the development and growth of prostate cancer utilized prostate cancer cell lines as models of human prostate cancer.

$P C-3$ epithelial cell line was established from a human metastatic to bone prostate adenocarcinoma. These cells are anchorage-dependent, morphologically similar to poorly differentiated adenocarcinomas, and produce tumors in nude mice. Additionally, these cells are characterized by features frequently observed in neoplastic cells, including abnormal nuclei and mitochondria [8].

DU145 cells were established from a prostate adenocarcinoma that was metastatic to the brain. When introduced into nude mice, these cells are capable of forming solid tumors. Furthermore, the tumors grown in mice are remarkably comparable to the original human tumor and tissue cultures $[9$, $10]$.

$L N C a P$ cell line was isolated in 1977 from a prostate adenocarcinoma metastatic to the left supraclavicular lymph node. These cells produce human prostatic acid phosphatase, prostate specific antigen, and androgen receptors. Furthermore, these cells are responsive to hormones such as $5 \alpha$-dihydrotestosterone and the frequency of tumor development correlates with hormonal levels $[11,12]$.

LNCaP-Al is an androgen-independent cell line derived from $\mathrm{LNCaP}$ cells. The androgen-independent cells were generated by continuously culturing LNCaP cells in an androgenfree medium. The surviving cells displayed phenotypic changes that included a morphology that differed from the LNCaP cells and androgen-independence [13].
$A R C a P$ prostate cancer cell line was established in 1996 from cells derived from the ascites fluid of a patient with metastatic prostate cancer. These cells express low levels of androgen receptor and prostate-specific antigen. Furthermore, their growth is suppressed by both estrogen and androgen in a dosedependent manner. Additionally, the cells are highly metastatic to sites including the lymph nodes, bones, lungs, and liver [14]. The increased growth and invasive nature of these cells directly correlate to epithelial-mesenchymal transition [15].

\section{Phytochemicals and Traditional Medicine}

There exists a plethora of evidence that supports the consumption of plant-derived foods for the prevention of chronic diseases. Utilization of these foods provides a wide variety of nutrients and phytochemicals that support normal growth and provide protection against the development of numerous chronic diseases. Identified phytochemicals are organized into the six major categories of phenolics, alkaloids, nitrogen-containing compounds, organosulfur compounds, phytosterols, and carotenoids [16]. Phenolics are produced by plants as secondary metabolites and have major protective roles. Interestingly, these roles include anticancer, anti-inflammatory, and antibacterial activities. To date, several thousand phenolic compounds have been isolated from a variety of plants including fruits, legumes, and cereals $[16,17]$. Plants produce alkaloids as secondary metabolites in response to stress. Cytotoxicity and antiinflammatory activity are among the identified properties of these compounds [18]. Isothiocyanates are among the most well-studied plant-derived organosulfur compounds. Importantly, this group of compounds has chemoprotective activity $[16,19]$. Both polyphenols and carotenoids are known to have antioxidant and anti-inflammatory activity. Additionally, these phytochemicals provide protection against oxidative stress associated with a number of chronic diseases including diabetic retinopathy and macular degeneration [16, 20]. Given their history of use in traditional medicines, and chemoprotective potential, phytochemicals present an exciting group of potential additional therapeutic reagents for preventing and treating cancers.

\section{Effects of Extracts from Specific Plant Families on Human Prostate Cancer Cells}

In recent years, important plant families have been analyzed for their potential to be used as anti-cancer treatments. The anti-cancer properties of these plants are directly linked to the bioactive compounds found in their extracts. While there are similarities in the bioactive compounds present in many of these plants, recent studies have identified unique components that may prove valuable as chemotherapeutic agents (Table 1). 


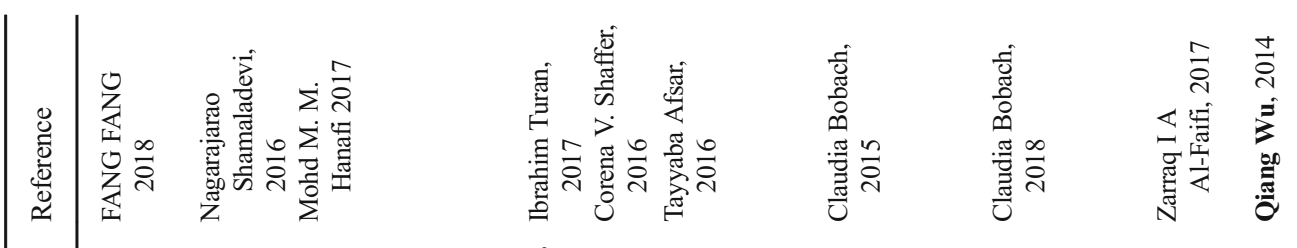

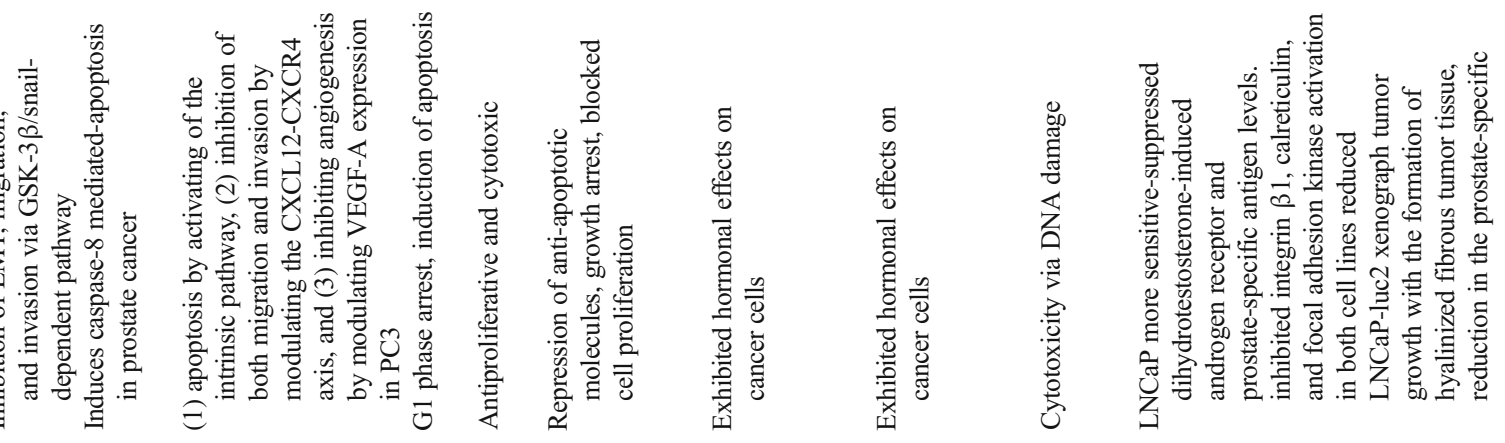

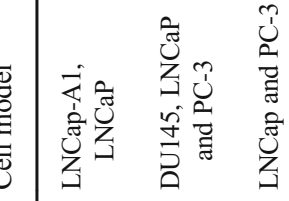

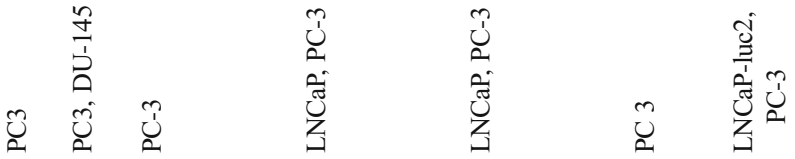

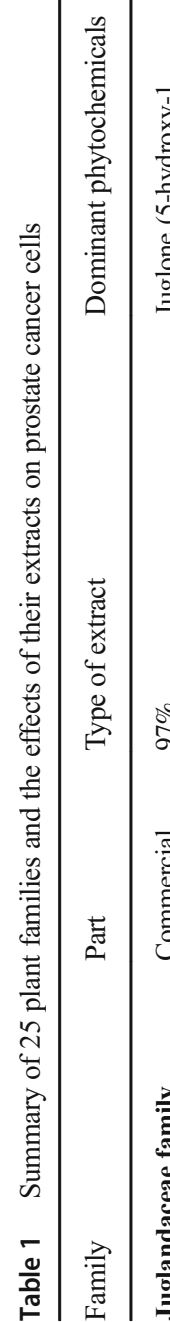
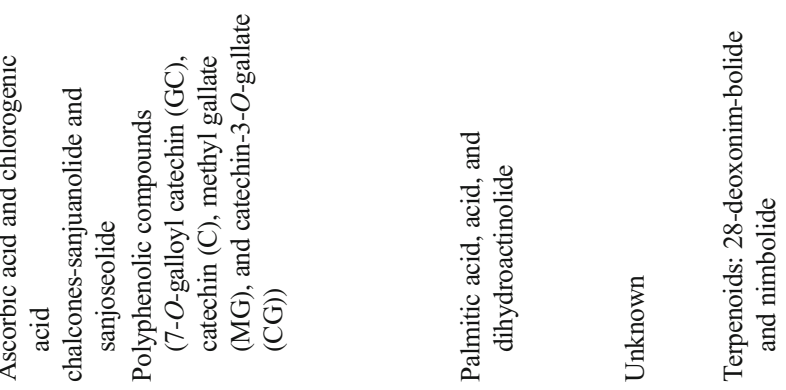

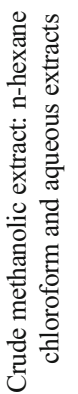

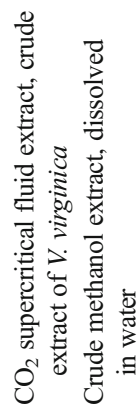

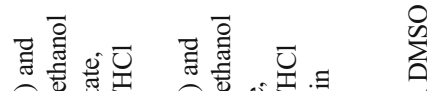

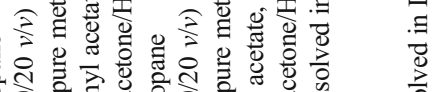

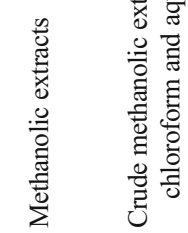

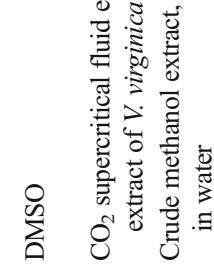

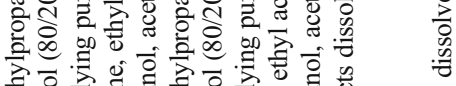

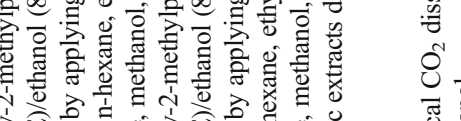

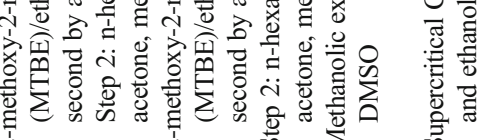

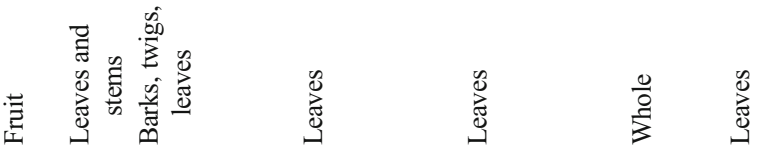

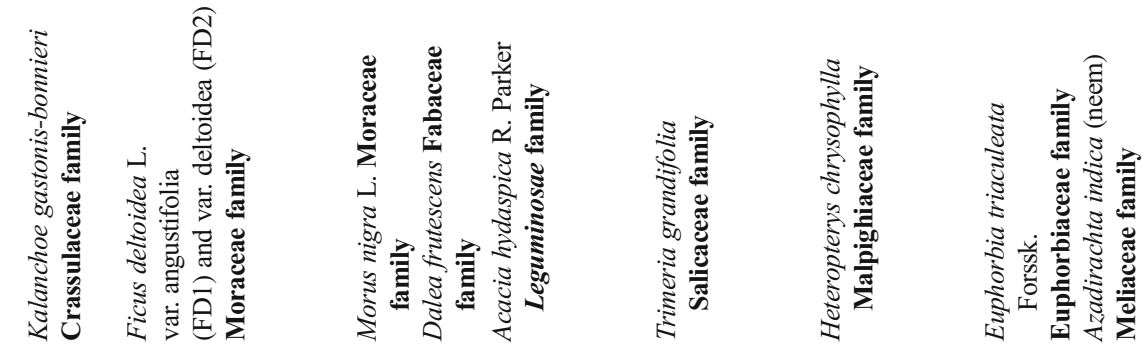




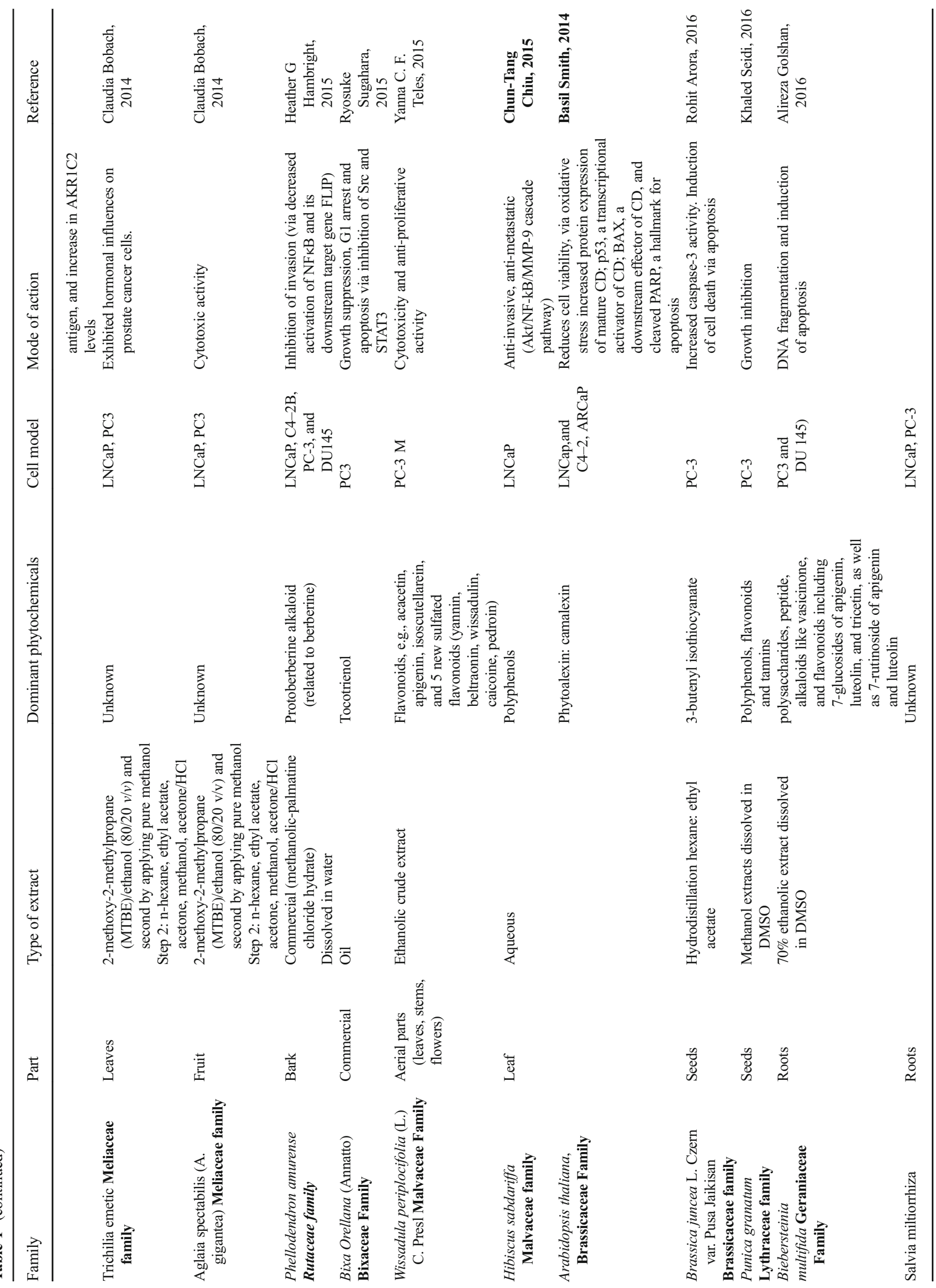




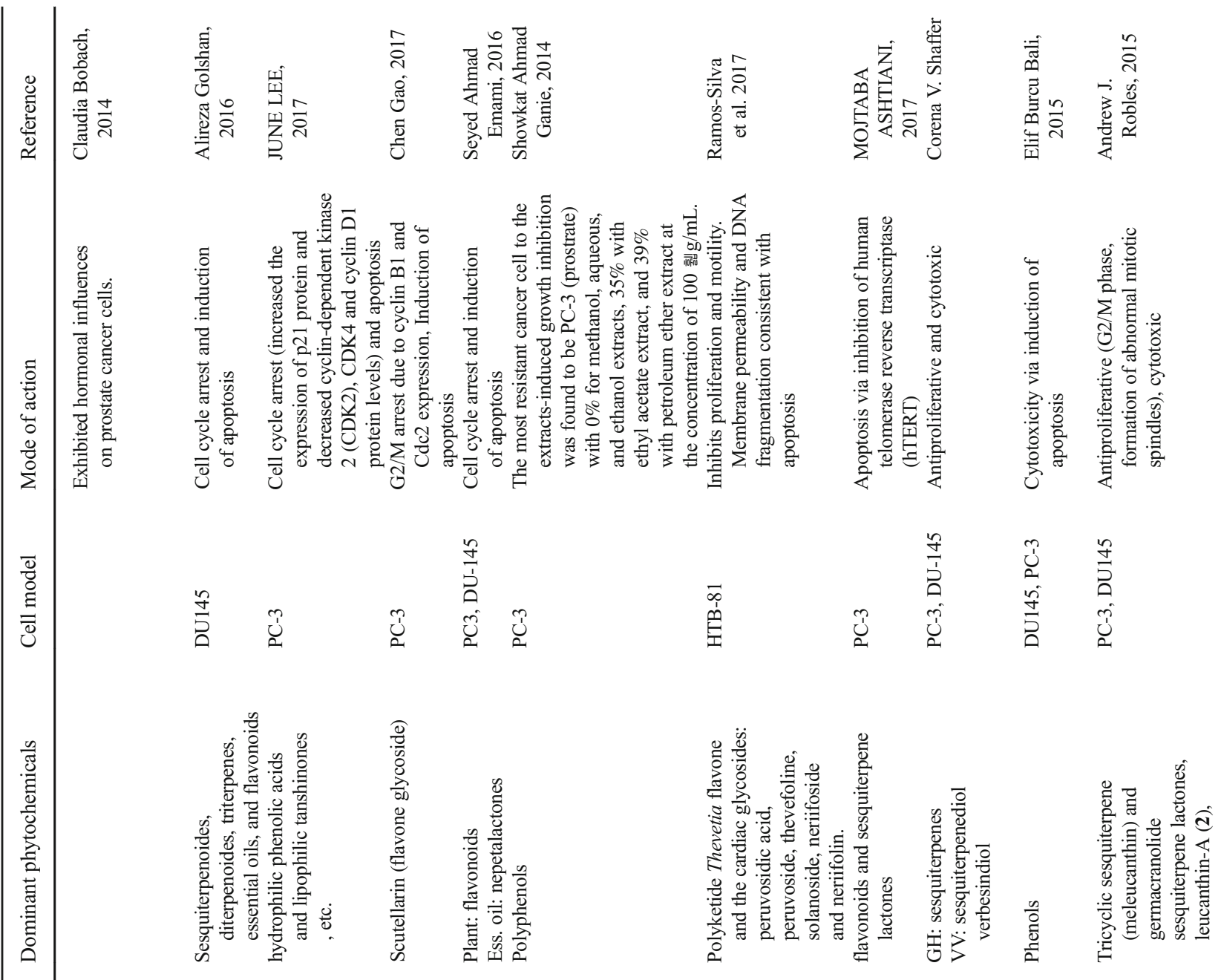

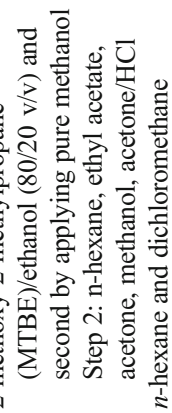

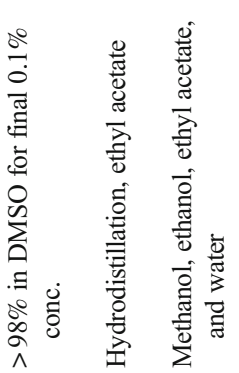

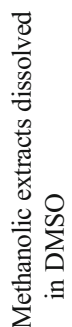

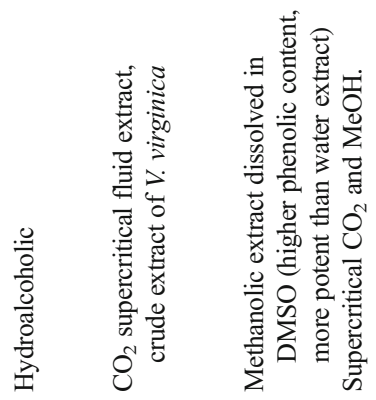

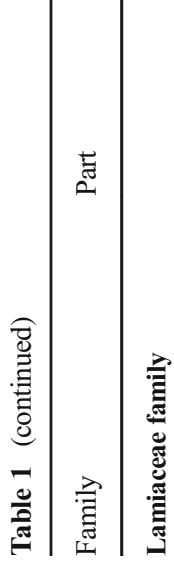

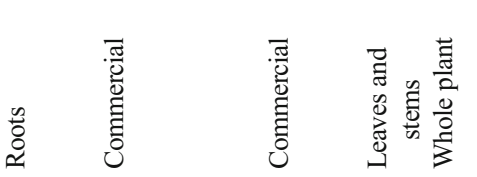

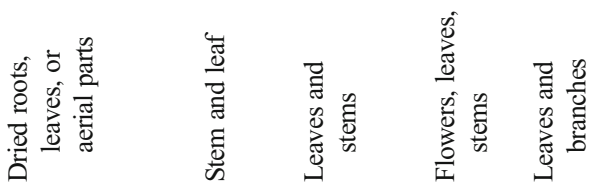

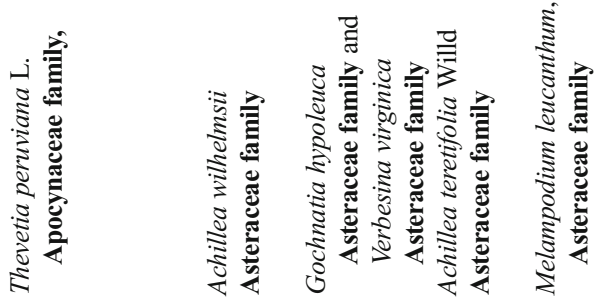




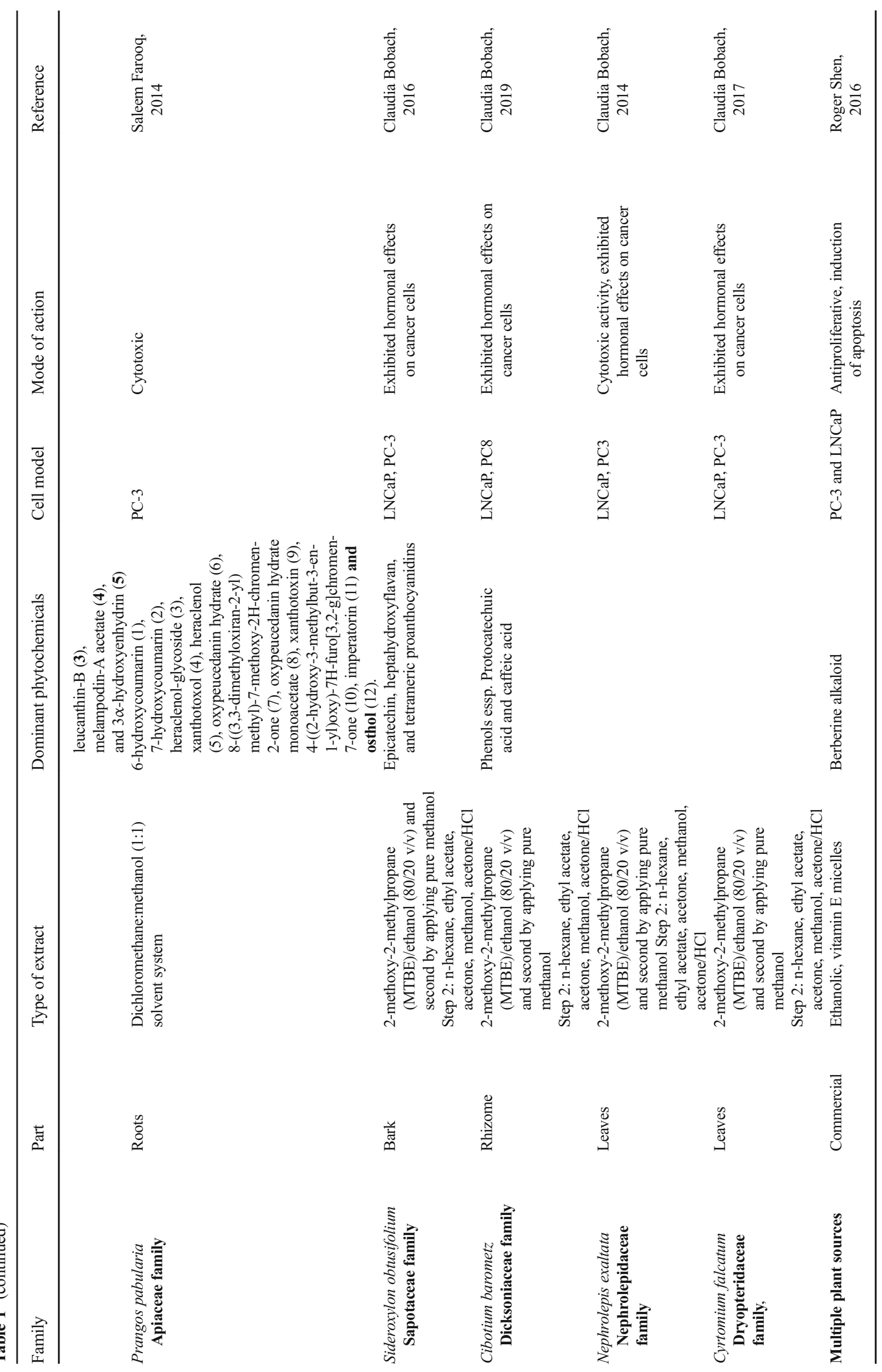


Juglandaceae The members of this family are distributed mainly in temperate zones. They are composed of trees and shrubs that include nut-producers such as walnut. Juglone is one of the most prevalent phytochemicals produced by members of this family. Juglone was shown to inhibit epithelial-mesenchymal transition (EMT), migration, and invasion of LNCaP and LNCap-A1 cells via the glycogen synthase kinase- $3 \beta /$ snail-dependent pathway [21]. EMT is of particular importance in the progression of cancers since epithelial cancer cells that transform to a mesenchymal phenotype tend to be more invasive and thus more prone to metastasis. Furthermore, since metastases that involve the bone drastically reduces the survival rate of patients [1], inhibition of this process presents a potent therapeutic target for impeding the progression of prostate and other cancers.

Crassulaceae Crassulaceae are perennial dicotyledons that can be either herbaceous, shrub-like, or tree-like. These plants are native to warm, dry regions of the world. Methanolic extract of Kalanchoe gastonis-bonnieri roots contains uronic acid. The induction of caspase-8-mediated apoptosis was observed in DU145, LNCaP, and PC-3 cells exposed to this extract results in [22]. Evasion of apoptosis is one of the classic features of cancer cells. Therefore, compounds that induce apoptosis in cancer cells are essential in hindering a major aspect of the cancer phenotype. Both caspase- 3 and caspase- 8 are regulators of programmed cell death. Interestingly, expression of caspase-3 has been previously shown to be associated with increased survival of patients with breast cancer. These observations strongly suggest that induction of caspase- 8 expression could potentially increase survival of patients with prostate cancer [23].

Moraceae The Moraceae family are native to tropical and subtropical regions and are either deciduous or evergreen varieties of trees and shrubs. Many genera are valued for their edible fruits while others produce waxy, latex materials. A variety of phytochemicals, including flavonoids, triterpenoids, ascorbic acid, and chlorogenic acid, have been isolated from members of the Moraceae family [24, 25]. Extracts from the angustifolia and deltoidea varieties of Ficus deltoidea induced apoptosis and inhibited invasion and migration of $\mathrm{LNCaP}$ and PC-3 cells. Furthermore, these plant extracts inhibited angiogenesis, a process that supports tumor formation, by modulating the expression of vascular endothelial growth factor-A in PC-3 cells [24]. Similarly, extracts from the fruit of Morus nigra, which contains high levels of ascorbic and chlorogenic acids, promoted cell cycle arrest in the Gap 1 phase and induced apoptosis in PC-3 cells [25]. Angiogenesis and uncontrolled growth are key features of cancer cells. Inhibition of either process therefore has the potential to limit the growth of cancer cells and tumor formation.
Leguminosae and Fabaceae Leguminosae and Fabaceae are used interchangeably to refer to a family of economically valuable flowering shrubs, trees, and herbaceous plants. Plants of this family are distributed worldwide with the exception of the Arctic and Antarctic regions. Methanolic extracts of the bark, twigs, and leaves of Acacia hydaspica contains polyphenolic compounds-7-O-galloyl catechin, catechin, methyl gallate, and catechin-3-O-gallate. These phytochemicals repressed the expression of anti-apoptotic molecules, and inhibited the proliferation, in PC-3 cells [26]. Similarly, extracts from the leaves and stems of Dalea frutescens, which contain chalconessanjuanolide and sanjoseolide, are anti-proliferative and cytotoxic to PC-3 and DU145 cells [27]. Together, these observations indicate that members of this family produce compounds with therapeutic potential as growth inhibitors. This is not entirely surprising since phenolic compounds are generally believed to have anti-cancer properties $[16,17]$.

Salicaceae This is a family of shrubs and trees that are either deciduous or evergreen. Their habitat is distributed worldwide, but they are most commonly found in tropical regions. Extracts from the leaves of Trimeria grandifolia inhibited the growth of LNCaP and PC-3 cancer cells [28]. Since LNCap cells rely on androgenic cues to promote growth, their inhibition by phytochemicals derived from this family suggests that these compounds have anti-androgenic properties. This ability confers the potential for these compounds to be used in hormonal therapy for prostate cancer.

Malpighiaceae This is a family of flowering plants that are native to tropical and subtropical regions of the world. The leaves of Heteropterys chrysophylla contain palmitic acid and dihydroactinolide. Extracts of these leaves also exhibited hormonal effects on LNCaP cells [28]. These observations, similar to those of Trimeria grandifolia-a member of the Salicaceae family, supports the potential for compounds from this plant to be used in hormonal therapy for prostate cancer.

Euphorbiaceae This a large family of flowering spurges, many of which are used as food sources. Well-known members include cassava and poinsettia. Methanolic extracts of Euphorbia triaculeata is cytotoxic to PC-3 cells via a mechanism that results in DNA damage and subsequent cell death [29]. Interestingly, the observed effects of the extracts were similar to those seen following exposure to doxorubicin, a well-known anti-cancer drug. However, unlike doxorubicin, the extract did not negatively impact normal cells. This selective toxicity, therefore, makes extracts of E. Triaculeata, a potential anti-cancer therapy that does not have the negative side effects observed with current treatments. 
Meliaceae This family is composed of flowering trees and shrubs that are native to tropical and subtropical regions. Several members are prized for their economically valuable timber, while others produce medicinal oils. Terpenoids are a phytochemical that is commonly found in these plants. Extract from the leaves of Azadirachta indica, commonly known as neem, contains the terpenoids, 28-deoxonimbolide, and nimbolide. Additionally, this extract inhibits integral $(\beta 1)$, calreticulin, and focal adhesion in LNCaP and PC-3 cells. Furthermore, the extract suppressed dihydrotestosteroneinduced androgen receptor and prostate-specific antigen levels in $\mathrm{LNCaP}$ cells and reduced the growth of LNCaP xenograph tumors [30]. Similarly, extracts from the leaves of Trichilia emetic also exhibit hormonal influences on LNCaP and PC3 cells. Extracts from the fruit of Aglaia spectabilis also show cytotoxic activity in PC-3 and LNCaP cells [28]. These data provide compelling evidence that extracts from the Meliaceae family of plants have the potential to be used in hormonal therapy and in the prevention and treatment of tumor growth.

Rutaceae This is a family of flowering, woody shrubs and trees that are widely distributed, but grow predominantly in the tropical and subtropical regions of the world. Berberine alkaloids are the most prevalent phytochemicals present in these plants [31]. The bark of Phellodendron amurense contains predominantly photoberberine. This phytochemical inhibited the invasion of LNCaP, C4-2B, DU145, and PC-3 cells via decreased activation of NF-kB [31]. Interestingly, vitamin $\mathrm{E}$ micelles of berberine exhibited anti-proliferative activity and promoted apoptosis in PC-3 and LNCap cells [32]. Metastasis of prostate cancers is associated with reduced survival rate [1]. Therefore, there is an urgency to identify compounds that inhibit this process.

Bixaceae A family of dicotyledonous trees, shrubs, and herbs. Several members produce annatto, a red pigment that is used in dyes and paints. Exposure of PC-3 cells to tocotrienol, a phytochemical isolated from the oil of Bixa orellana, results in growth suppression. Furthermore, the cells arrest in the G1 phase of the cell cycle and subsequently undergo apoptosis via the inhibition of Src and STAT3 [33]. Deregulation of the expression of Src and STAT has been implicated in other cancers. Moreover, in the murine model, inhibition of Src2 is directly linked to the suppression of prostate cancer growth and metastasis [34]. Together, these data provide strong support for the potential use of these compounds in the treatment of human cancers.

Malvaceae These plants include shrubs, herbs, and trees that grow predominantly in tropical regions. Some members of this family, including Theobroma cacao from which chocolate is made, are prized cash crops. The phytochemicals identified in members of this family include flavonoids and polyphenols, both of which have been shown to have anticancer and antiinflammatory properties [16, 35, 36]. Five novel sulfate flavonoids, yannin, beltraonin, wissadulin, caicoine, and pedroin, were isolated from extracts derived from the aerial parts of Wissadula periplocifolia. This extract was both cytotoxic and anti-proliferative to PC-3 cells [36]. On the other hand, extracts derived from the leaves of Hibiscus sabdariffa, which contains mainly polyphenols, exhibited anti-invasive and antimetastatic activity in LNCaP cells via the Akt/NF-kB/MMP9 cascade pathway [35]. Uncontrolled growth is a hallmark of cancer cells, while metastases frequently occur with very aggressive cancers and severely reduces the survival rate of patients [1]. Members of this family produce phytochemicals capable of targeting both growth and invasion, thus making them a unique candidate for bioprospecting.

Brassicaceae This is a family of economically important flowering plants that was formerly classified as Cruciferae and includes edible plants such as cabbage and broccoli. Phytoalexins and isothiocyanate are among some of the phytochemicals produced by members of this family. Specifically, extracts of Arabidopsis thaliana contain camalexin, a type of phytoalexin. This extract reduces the viability of LNCaP, C42 , and ARCaP prostate cancer cells via increasing oxidative stress. Specifically, exposure causes increased expression of p53, BAX, and PARP, proteins that are all involved in apoptosis [37]. Apoptosis is also induced in PC-3 cells by extract derived from the seed of Brassica juncea var. Pusa Jaikisan, which contains 3-butenyl isothiocyanate. However, in the latter study, an increase in caspase-3 activity was also observed in PC-3 cells [38].

Lythraceae This family of flowering herbs, shrubs, and trees are distributed worldwide. However, the majority of species are native to tropical and subtropical regions. The seeds of Punica granatum, commonly known as pomegranate, contains large quantities of polyphenols, which are anti-inflammatory, flavonoids, and tannins [16]. As would be expected based on the presence of polyphenols, this extract inhibits the growth of PC-3 cells [39]. The specific mechanism by which this growth inhibition occurs has not yet been elucidated. However, it likely involves apoptosis as is induced by other plant families, such a Moraceae and Geraniaceae, which contain similar phytochemicals [25, 40].

Geraniaceae These are dicotyledonous flowering shrubs that are native to temperate regions of the world. Ethanolic extract of the roots of Biebersteinia multifida contains polysaccharides, peptide, alkaloids, and flavonoids including 7glucosides of apigenin, luteolin, and tricetin, 7-rutinoside of apigenin, and luteolin. Exposure of PC-3 and DU145 cells to this extract results in DNA fragmentation and the induction of apoptosis [40]. While DNA fragmentation may occur 
spontaneously, it is also a key feature of apoptosis, and therefore an important chemotherapeutic target.

Lamiaceae Plants in this family are flowering aromatics that are native to temperate regions throughout the world. Most members are perennial and annual herbs, prized for their flowers and scented leaves. Prominent constituents of this family include flavonoids, phenolic acids, lipophilic tanshinones, sesquiterpenoids, diterpenoids, and triterpenoids. Nepeta cataria, Scutellaria altissima, Salvia miltiorrhiza, Salvia chorassanica, and Salvia miltiorrhiza, all members of the Lamiaceae family exhibited cytotoxic effects on DU145, PC-3, or LNCaP prostate cancer cells and induced cell cycle arrest and subsequent apoptosis via a variety of mechanisms [28, 41-44]. PC-3 cells treated with extract from Scutellaria altissima, which contains predominantly scutellarin, a flavone glycoside, arrest in the GAP2/mitotic phase as a result of the increased expression of cyclin B1 and $\mathrm{Cdc} 2$ [43]. Similarly, PC-3 cells treated with acetonitrile extract of Salvia miltiorrhiza show cell cycle arrest due to increased expression of $\mathrm{p} 21$, and decreased expression of cyclin-dependent kinases 2 and 4, and cyclin D1 [44]. Salvia miltiorrhiza contains hydrophilic phenolic acids and lipophilic tanshinones among other phytochemicals [41]. Interestingly, this plant also exhibits hormonal influences on LNCap and PC-3 cancer cells, which suggests it has potential to be used in hormonal therapy of prostate cancer [28].

Boraginaceae This family is made up of flowering trees, herbs, and shrubs that are either annuals or perennials. While a large number of species are ornamentals, some members are poisonous to humans and animals. Methanolic extracts of Arnebia benthamii contains polyphenols and inhibits the growth of PC-3 cells [45]. Polyphenols are known to have antioxidant and anti-inflammatory activity $[16,17]$. These data indicate that polyphenols are also cytotoxic to cancer cells and are therefore a potential therapeutic compound.

Apocynaceae This is a family of flowering trees, shrubs, herbs, and vines that are native to tropical and subtropical regions of the world. While many members contain poisonous alkaloids, others are valued ornamentals. Methanolic extract of the aerial parts of Thevetia peruviana contains polyketide Thevetia flavoneperuvosidic acid, peruvoside, thevefoline, solanoside, neriifoside, and neriifolin. This extract induced apoptosis in HTB-81 (DU145) prostate cancer adenocarcinoma via inhibition of cell-proliferation and motility, and induction of membrane permeability and DNA fragmentation [46]. DNA fragmentation is also induced by Biebersteinia multifida, a member of the Geraniaceae family that also produces flavonoids [40]. These observations suggest that flavonoids are responsible for DNA fragmentation and the subsequent induction of apoptosis in prostate cancer cells.
Asteraceae Members of this family tend to be flowering herbs, shrubs, or trees, and are distributed throughout the world. Many members are traditionally used as ornamental plants or food sources. Flavonoids, sesquiterpenes, and phenols are frequently isolated phytochemicals from plants in this family. Extracts derived from Achillea wilhelmsii, Gochnatia hypoleuca, Verbesina virginica, Achillea teretifolia Willd, and Melampodium leucanthum have anti-proliferative and cytotoxic activity against PC-3 and DU145 prostate cancer cell lines [27, 47-49]. In the case of Achillea wilhelmsii, whose main bioactive compounds are flavonoids and sesquiterpenes lactones, cytotoxicity is based on the induction of apoptosis via the inhibition of the enzyme telomerase reverse transcriptase [49]. Melampodium leucanthum, on the other hand, contains tricyclic sesquiterpenes and germacranolide sesquiterpene lactones that induces death of PC-3 and DU145 cells by causing them to arrest in the GAP2/mitotic phase of cells division and by the formation of abnormal mitotic spindle [48]. This family appears to have several potential chemotherapeutic mechanisms, making it a leading candidate for further investigation.

Apiaceae Carrot and celery are well-known edible members of this large family of annual, perennial, and biennial flowering herbs, shrubs, or small trees. Extracts from the roots of Prangos pabularia contains a variety of phytochemicals, including 6-hydroxycoumarin, 7-hydroxycoumarin, heraclenolglycoside, xanthotoxol, heraclenol, oxypeucedanin hydrate, and osthol, which are cytotoxic to PC-3 cells [49]. Although the exact mechanism of cytotoxicity has not yet been elucidated, it can be assumed that it results in the induction of apoptosis, a desired property of chemotherapeutic compounds.

\section{Sapotaceae, Dicksoniaceae, Nephrolepidaceae, Dryopteridaceae} Sapotaceae are a family of evergreen, flowering trees, and shrubs that grow mainly in the tropics. Extracts from the bark of Sideroxylon obtusifolium contains a variety of phytochemicals including epicatechin, heptahydroxyflavan, and tetrameric proanthocyanidins. These extracts elicited hormonal effects on PC-3 and LNCaP cells [28].

The Dicksoniaceae family of ferns that are native to warm tropics, subtropics, and temperate regions of the world. Extracts of the rhizome of Cibotium barometz contains the phenols protocatechuic acid and caffeic acid. These extracts induce hormonal effects on LNCaP and PC-3 cells [28].

Nephrolepidaceae, also called Dryopteridaceae, includes plants commonly known as ferns. Although widely distributed, they predominantly grow in tropical and temperate regions of the world. Extract from the leaves of Nephrolepis exaltata is cytotoxic to PC-3 and LNCaP cells. Furthermore, this extract elicits hormonal effects on these cells. Similar effects were observed when these cells were treated with the leaf extract of Cyrtomium falcatum, another member of this family [28]. 
Extracts from the Cyrtomium falcatum species of these ferns exhibit hormonal effects on PC-3 and LNCap prostate cancer cells [28]. Extracts derived from members of Sapotaceae, Dicksoniaceae, Nephrolepidaceae, and Dryopteridaceae all elicited hormonal effects on a variety of prostate cancer models [28]. The specific pathways and mechanisms through which these families elicit their effects are unknown, these observations provide strong evidence their phytochemicals may be useful in the development of new prostate cancer therapies.

\section{Summary}

The twenty-four distinct plant families discussed in this review are categorized into three monophyletic groups, rosids, astrids, and pteridophytes, all of which fall under the angiosperm clade (Fig. 1). Angiosperms are a highly diverse clade of flowering plants that include more than 200,000 species. Interestingly, the species are not evenly distributed. In fact, several families contain a strikingly large number of members compared with other plant families [50].
The phytochemicals isolated from the species within the angiosperm clade appear to be as diverse as the clade itself (Table 1). As such, there appears to be no substantial distinction between the major categories of phytochemicals isolated from each family. This observation supports previous reports which indicated that phytochemicals such as phenols are produced by a wide variety of plants [17]. This is most likely due to the important protective role of these compounds in their organisms of origin. Although not well understood, the mechanisms of cytotoxicity of the reported plant compounds seem to be based on cell cycle arrest, the induction of apoptosis, and in a few cases, hormonal control that leads to reduced cell proliferation or death $[28,30]$. Furthermore, these different mechanisms are dispersed among the families (Table 1, Fig. 1).

Diets deficient in plant-based components have been identified as a possible major contributor to the development of cancer in the USA $[2,3]$. Many angiosperms which produce compounds that are cytotoxic to prostate cancer cells are valued food sources in other parts of the world. This suggests that these plant products may be a viable option for chemo-protection and chemotherapy. Moreover, it is worth investigating if simply

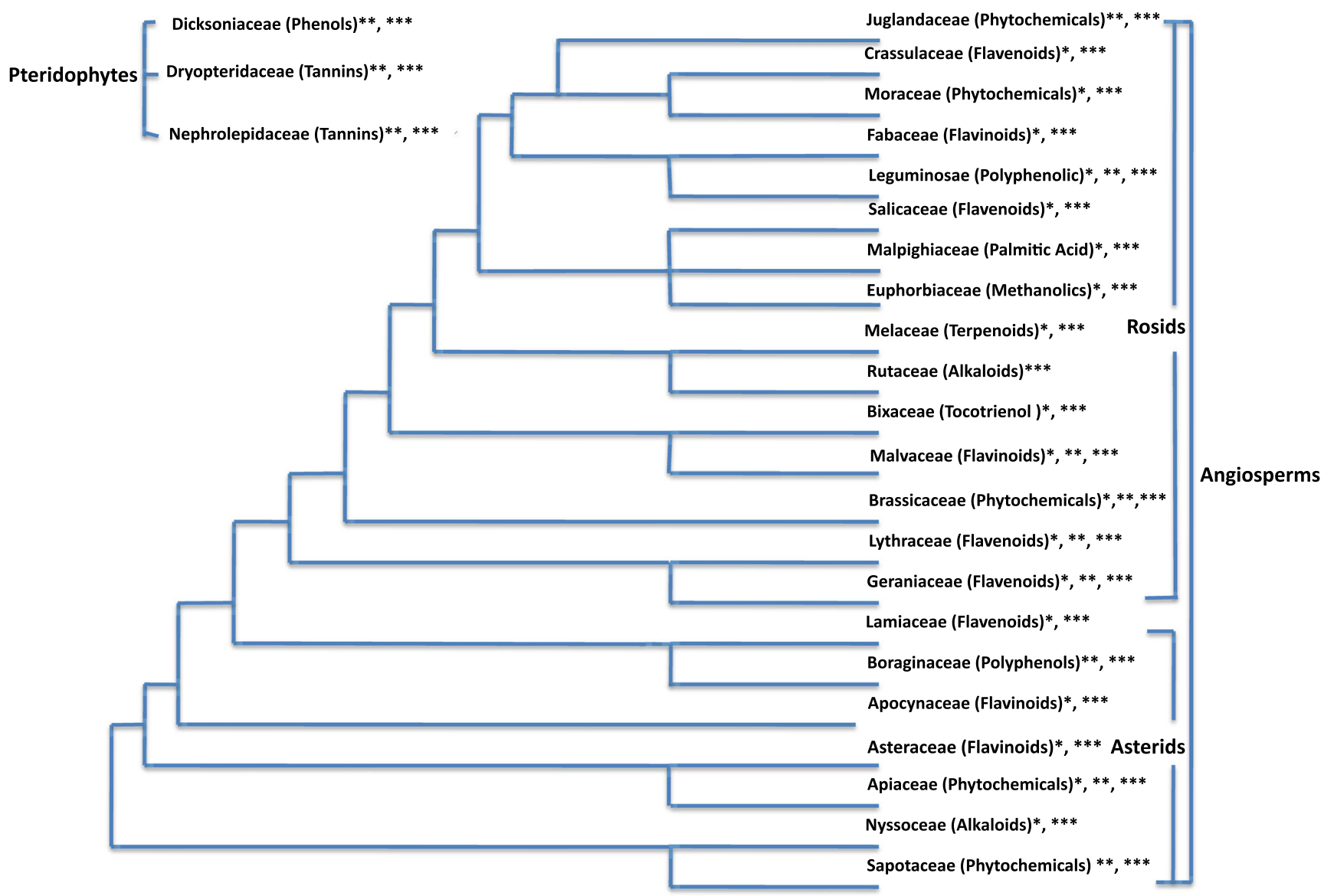

Fig. 1 Phylogenetic tree of the evolutionary relationship between medicinal plants and their plant-derived anti-carcinogenic bioactive compounds. Phylogenetic tree summarizes the plant-derived anti-carcinogenic bioactive compounds and their therapeutic effect. Asterisk illustrates anti-carcinogenic properties for each plant species. * Anti-inflammatory properties, $* *$ free radical scavenger/anti-oxidant, and $* * *$ antiproliferative properties 
modifying the diets of individuals most likely to develop cancer to include more of these beneficial foods is sufficient to provide significant protection or even chemotherapy. A positive outcome of this approach could change the landscape of health and medicine as we know it.

Given their varied mechanisms of action, phytochemicals have the potential to be potent chemotherapeutics that are less costly and have fewer harmful side effects when compared to the drugs currently used to treat prostate cancer.

\section{Compliance with Ethical Standards}

Conflict of Interest Cindy Thomas-Charles and Herman Fennell declare no potential conflict of interest.

Human and Animal Rights and Informed Consent This article does not contain any studies with human or animal subjects performed by any of the authors.

Open Access This article is distributed under the terms of the Creative Commons Attribution 4.0 International License (http:// creativecommons.org/licenses/by/4.0/), which permits unrestricted use, distribution, and reproduction in any medium, provided you give appropriate credit to the original author(s) and the source, provide a link to the Creative Commons license, and indicate if changes were made.

\section{References}

1. Gutierrez-Uzquiza A, Lopez-Haber C, Jernigan DL, Fatatis A, Kazanietz MG. PKCE is an essential mediator of prostate cancer bone metastasis. Mol Cancer Res. 2015;13(9):1336-46.

2. Shuch B, Mikhail M, Satagopan J, Lee P, Yee H, Chang C, et al. Racial disparity of epidermal growth factor receptor expression in prostate cancer. J Clin Oncol. 2004;22(23):4725-9.

3. Bhardwaj A, et al. Racial disparities in prostate cancer: a molecular perspective. Front Biosci (Landmark Ed). 2017;22:772-82.

4. Willett WC. Diet, nutrition, and avoidable cancer. Environ Health Perspect. 1995;103(Suppl 8):165-70.

5. Jordan MA, Wilson L. Microtubules as a target for anticancer drugs. Nat Rev Cancer. 2004;4(4):253-65.

6. Abu Saleh M, et al. Inhibition of DNA topoisomerase type IIalpha (TOP2A) by mitoxantrone and its halogenated derivatives: a combined density functional and molecular docking study. Biomed Res Int. 2016;2016:6817502.

7. Wei C, Pan Y, Huang H, Li YP. Estramustine phosphate induces prostate cancer cell line PC3 apoptosis by down-regulating miR-31 levels. Eur Rev Med Pharmacol Sci. 2018;22(1):40-5.

8. Kaighn ME, et al. Establishment and characterization of a human prostatic carcinoma cell line (PC-3). Investig Urol. 1979;17(1):1623.

9. Mickey DD, Stone KR, Wunderli H, Mickey GH, Vollmer RT, Paulson DF. Heterotransplantation of a human prostatic adenocarcinoma cell line in nude mice. Cancer Res. 1977;37(11):4049-58.

10. Stone KR, Mickey DD, Wunderli H, Mickey GH, Paulson DF. Isolation of a human prostate carcinoma cell line (DU 145). Int J Cancer. 1978;21(3):274-81.

11. Horoszewicz JS, et al. The LNCaP cell line-a new model for studies on human prostatic carcinoma. Prog Clin Biol Res. 1980;37:11532 .
12. Horoszewicz JS, Leong SS, Kawinski E, Karr JP, Rosenthal H, Chu $\mathrm{TM}$, et al. LNCaP model of human prostatic carcinoma. Cancer Res. 1983;43(4):1809-18.

13. Xu G, Wu J, Zhou LL, Chen B, Sun Z, Zhao F, et al. Characterization of the small RNA transcriptomes of androgen dependent and independent prostate cancer cell line by deep sequencing. PLoS One. 2010;5(11):e15519.

14. Zhau HY, et al. Androgen-repressed phenotype in human prostate cancer. Proc Natl Acad Sci U S A. 1996;93(26):15152-7.

15. Xu J, Wang R, Xie ZH, Odero-Marah V, Pathak S, Multani A, et al. Prostate cancer metastasis: role of the host microenvironment in promoting epithelial to mesenchymal transition and increased bone and adrenal gland metastasis. Prostate. 2006;66(15):1664-73.

16. Liu RH. Health-promoting components of fruits and vegetables in the diet. Adv Nutr. 2013;4(3):384s-92s.

17. Shahidi, F. and J. Yeo, Bioactivities of phenolics by focusing on suppression of chronic diseases: a review. Int J Mol Sci, 2018. 19(6).

18. Peng J, Zheng TT, Li X, Liang Y, Wang LJ, Huang YC, et al. Plantderived alkaloids: the promising disease-modifying agents for inflammatory bowel disease. Front Pharmacol. 2019;10:351.

19. Dinkova-Kostova AT. Chemoprotection against cancer by isothiocyanates: a focus on the animal models and the protective mechanisms. Top Curr Chem. 2013;329:179-201.

20. Bungau S, et al. Health benefits of polyphenols and carotenoids in age-related eye diseases. Oxidative Med Cell Longev. 2019;2019: 9783429.

21. Fang F, Chen S, Ma J, Cui J, Li Q, Meng G, et al. Juglone suppresses epithelial-mesenchymal transition in prostate cancer cells via the protein kinase $\mathrm{B} / \mathrm{glycogen}$ synthase kinase-3beta/snail signaling pathway. Oncol Lett. 2018;16(2):2579-84.

22. Shamaladevi N, Araki S, Lyn DA, Ayyathurai R, Gao J, Lokeshwar $\mathrm{VB}$, et al. The andean anticancer herbal product BIRM causes destabilization of androgen receptor and induces caspase- 8 mediatedapoptosis in prostate cancer. Oncotarget. 2016;7(51):84201-13.

23. Pu X, Storr SJ, Zhang Y, Rakha EA, Green AR, Ellis IO, et al. Caspase- 3 and caspase- 8 expression in breast cancer: caspase- 3 is associated with survival. Apoptosis. 2017;22(3):357-68.

24. Hanafi, M.M.M., et al., In vitro pro-apoptotic and anti-migratory effects of Ficus deltoidea L. Plant extracts on the human prostate cancer cell lines PC3. Front Pharmacol, 2017. 8: p. 895.

25. Turan I, Demir S, Kilinc K, Burnaz NA, Yaman SO, Akbulut K, et al. Antiproliferative and apoptotic effect of Morus nigra extract on human prostate cancer cells. Saudi Pharm J. 2017;25(2):241-8.

26. Afsar T, Trembley JH, Salomon CE, Razak S, Khan MR, Ahmed K. Growth inhibition and apoptosis in cancer cells induced by polyphenolic compounds of Acacia hydaspica: involvement of multiple signal transduction pathways. Sci Rep. 2016;6:23077.

27. Shaffer CV, Cai S, Peng J, Robles AJ, Hartley RM, Powell DR, et al. Texas native plants yield compounds with cytotoxic activities against prostate cancer cells. J Nat Prod. 2016;79(3):531-40.

28. Bobach C, Schurwanz J, Franke K, Denkert A, Sung TV, Kuster R, et al. Multiple readout assay for hormonal (androgenic and antiandrogenic) and cytotoxic activity of plant and fungal extracts based on differential prostate cancer cell line behavior. J Ethnopharmacol. 2014;155(1):721-30.

29. Al-Faifi, Z.I., et al., Evaluation of cytotoxic and genotoxic effects of Euphorbia triaculeata Forssk. extract Asian Pac J Cancer Prev, 2017. 18(3): p. 771-777.

30. Wu Q, Kohli M, Bergen HR III, Cheville JC, Karnes RJ, Cao H, et al. Preclinical evaluation of the supercritical extract of Azadirachta indica (neem) leaves in vitro and in vivo on inhibition of prostate cancer tumor growth. Mol Cancer Ther. 2014;13(5): 1067-77. 
31. Hambright HG, Batth IS, Xie J, Ghosh R, Kumar AP. Palmatine inhibits growth and invasion in prostate cancer cell: potential role for rpS6/NFkappaB/FLIP. Mol Carcinog. 2015;54(10):1227-34.

32. Shen R, Kim JJ, Yao M, Elbayoumi TA. Development and evaluation of vitamin E d-alpha-tocopheryl polyethylene glycol 1000 succinate-mixed polymeric phospholipid micelles of berberine as an anticancer nanopharmaceutical. Int J Nanomedicine. 2016;11: $1687-700$.

33. Sugahara R, et al. Annatto tocotrienol induces a cytotoxic effect on human prostate cancer PC3 cells via the simultaneous inhibition of Src and Stat3. J Nutr Sci Vitaminol (Tokyo). 2015;61(6):497-501.

34. Dasgupta, S., Putluri N., Long W., Zhang B., Wang J., Kaushik A. K., Arnold J. M., Bhowmik S. K., Stashi E., Brennan C. A., Rajapakshe K., Coarfa C., Mitsiades N., Ittmann M. M., Chinnaiyan A. M., Sreekumar A., O'Malley B. W., Coactivator SRC-2-dependent metabolic reprogramming mediates prostate cancer survival and metastasis. J Clin Invest, 2015. 125(3): p1174-88, 1174.

35. Chiu CT, Chen JH, Chou FP, Lin HH. Hibiscus sabdariffa leaf extract inhibits human prostate cancer cell invasion via downregulation of Akt/NF-kB/MMP-9 pathway. Nutrients. 2015;7(7): 5065-87.

36. Teles YC, et al. New sulphated flavonoids from Wissadula periplocifolia (L.) C. Presl (Malvaceae). Molecules. 2015;20(11): 20161-72.

37. Smith B, Randle D, Mezencev R, Thomas LS, Hinton C, OderoMarah V. Camalexin-induced apoptosis in prostate cancer cells involves alterations of expression and activity of lysosomal protease cathepsin D. Molecules. 2014;19(4):3988-4005.

38. Arora R, Kumar R, Mahajan J, Vig AP, Singh B, Singh B, et al. 3Butenyl isothiocyanate: a hydrolytic product of glucosinolate as a potential cytotoxic agent against human cancer cell lines. J Food Sci Technol. 2016;53(9):3437-45.

39. Seidi K, Jahanban-Esfahlan R, Abasi M, Abbasi MM. Anti tumoral properties of Punica granatum (pomegranate) seed extract in different human cancer cells. Asian Pac J Cancer Prev. 2016;17(3):111922.

40. Golshan A, Hassanzadeh S, Mojdekanloo M, Tayarani-Najaran Z. Effects of Biebersteinia multifida hydro-ethanol extract on proliferation and apoptosis of human prostate cancer and human embryonic kidney cells. Avicenna. J Phytomed. 2016;6(6):671-7.

41. Emami SA, Asili J, HosseinNia S, Yazdian-Robati R, Sahranavard M, Tayarani-Najaran Z. Growth inhibition and apoptosis induction of essential oils and extracts of Nepeta cataria L. on human prostatic and breast cancer cell lines. Asian Pac J Cancer Prev. 2016;17(S3): 125-30.

42. Golshan A, Amini E, Emami SA, Asili J, Jalali Z, Sabouri-Rad S, et al. Cytotoxic evaluation of different fractions of Salvia chorassanica Bunge on MCF-7 and DU 145 cell lines. Res Pharm Sci. 2016;11(1):73-80.

43. Gao C, Zhou Y, Jiang Z, Zhao Y, Zhang D, Cong X, et al. Cytotoxic and chemosensitization effects of Scutellarin from traditional Chinese herb Scutellaria altissima L. in human prostate cancer cells. Oncol Rep. 2017;38(3):1491-9.

44. Lee J, Choi BY, Keum YS. Acetonitrile extract of Salvia miltiorrhiza Radix exhibits growth-inhibitory effects on prostate cancer cells through the induction of cell cycle arrest and apoptosis. Oncol Lett. 2017;13(5):2921-8.

45. Ganie, S.A., et al., In vitro antioxidant and cytotoxic activities of Arnebia benthamii (Wall ex. G. Don): a critically endangered medicinal plant of Kashmir Valley. Oxidative Med Cell Longev, 2014. 2014: p. 792574.

46. Ramos-Silva A, Tavares-Carreón F, Figueroa M, de la Torre-Zavala S, Gastelum-Arellanez A, Rodríguez-García A, et al. Anticancer potential of Thevetia peruviana fruit methanolic extract. BMC Complement Altern Med. 2017;17(1):241.

47. Bali EB, Açık L, Elçi P, Sarper M, Avcu F, Vural M. In vitro antioxidant, cytotoxic and pro-apoptotic effects of Achillea teretifolia Willd extracts on human prostate cancer cell lines. Pharmacogn Mag. 2015;11(Suppl 2):S308-15.

48. Robles AJ, Peng J, Hartley RM, Lee B, Mooberry SL. Melampodium leucanthum, a source of cytotoxic sesquiterpenes with antimitotic activities. J Nat Prod. 2015;78(3):388-95.

49. Ashtiani M, Nabatchian F, Galavi HR, Saravani R, FarajianMashhadi F, Salimi S. Effect of Achillea wilhelmsii extract on expression of the human telomerase reverse transcriptase mRNA in the PC3 prostate cancer cell line. Biomed Rep. 2017;7(3):251-6.

50. Farooq S, Shakeel-u-Rehman, Dangroo NA, Priya D, Banday JA, Sangwan PL, et al. Isolation, cytotoxicity evaluation and HPLCquantification of the chemical constituents from Prangos pabularia. PLoS One. 2014;9(10):e108713.

Publisher's Note Springer Nature remains neutral with regard to jurisdictional claims in published maps and institutional affiliations. 